\title{
New Stability Analysis for Systems with Interval Time-varying Delay via a Delay-fractioning Approach
}

\author{
Yuzhi Liu ${ }^{1,2}$ and Muguo $\mathrm{Li}^{2}$ \\ ${ }^{1}$ Faculty of Electronic Information and Electrical Engineering, Dalian University of \\ Technology, Dalian 116023, China \\ ${ }^{2}$ State Key Laboratory of Coastal and Offshore Engineering, Dalian University of \\ Technology, Dalian 116023, China \\ liuyuzhi_1@163.com,lmguo@dlut.edu.cn
}

\begin{abstract}
This paper addresses the problem of stability analysis for linear systems with interval timevarying delay. A general form of the delay-fractioning approach is proposed, which not only takes advantage of all possible information on the delay's lower bound, but also exploits further information between the delay's upper and lower bounds. A new Lyapunov-Krasovskii functional $(L K F)$ is constructed and delay-dependent stability criteria are derived in terms of linear matrix inequalities (LMIs) by using the piecewise analysis method. The convexity of the matrix function is used to avoid the conservatism caused by enlarging the time-varying delay to its upper bound in each subinterval. Numerical examples are given finally to verify the effectiveness of the proposed criteria.
\end{abstract}

Keywords: Time-varying delay, Lyapunov-Krasovskii functional (LKF), linear matrix inequality (LMI), delay-fractioning approach

\section{Introduction}

Time-delays are often encountered in various practical systems such as biological systems, social systems, chemical processes and networked control systems. Due to the presence of time-delays, instantaneous information cannot be available for control actions, which may lead to oscillation or instability. Therefore, many researchers have been devoted to investigating the stability of time-delay systems $[1,2,3]$.

The time-delay system's future evolution depends not only on its present state, but also on a period of its history. This cause-effect relationship has been traditionally modeled by functional differential equations. Usually, two approaches are used for stability analysis of time-delay systems: Razumikhin Theorem approach and Lyapunov-Krasovskii functional (LKF) approach. The latter approach is known to be less conservative for taking advantage of additional information of the time delay [1]. Two important sources of conservatism in the LKF approach are the choice of an appropriate LKF $V$ and the way to bound some cross terms arisen when manipulating the derivative of $V$. In the past decade, many methods were developed to reduce the conservatism of the LKF approach. For example, the descriptor model transformation method was used in [2] for studying the $\mathrm{H}_{1}$ control problem of linear time-delay systems. In [4, 5], the free weighting matrices were adopted to derive delaydependent stability criteria for systems with time-varying delay. In [6], a new stability analysis based on the Wirtinger's inequality was applied to sampled-data state-feedback 
stabilization and to a static output-feedback problem. In [7], the improved bounding technique was used for stability analysis of uncertain neutral systems with multiple time-varying delays.

Recently, a delay-fractioning approach for investigating stability of systems with constant time delay was presented in [8], and was extended to systems with time-varying delays in [9, $10,11,12]$. The delay-fractioning approach provides an efficient way to reduce conservatism for exploiting more information of the delay. In $[9,11]$, in order to reduce the conservatism of the delay-dependent stability criteria, the delay range $\left[\tau_{m}, \tau_{M}\right]$ was divided into two equally spaced subintervals: $\left[\tau_{m}, \tau_{a}\right]$ and $\left[\tau_{a}, \tau_{M}\right]$, where $\tau_{a}=\left(\tau_{m}+\tau_{M}\right) / 2$. In [10], the equal division idea of the delay range $\left[\tau_{m}, \tau_{M}\right]$ was applied to the stability analysis of networked control systems. In [12], the information of the delay's lower bound was further exploited through the partitioning of the delay interval $\left[0, \tau_{m}\right]$ into $\eta>0$ equally spaced subintervals, then better results were obtained, especially for larger values of $\tau_{m}$. However, in the existing literature, the information between the delay range $\left[\tau_{m}, \tau_{M}\right]$ was just exploited by halving idea, therefore, there is still room for improvement of the delay fractioning approach by exploiting further information in the delay range $\left[\tau_{m}, \tau_{M}\right]$, which motivates our present work.

In this paper, the interval $\left[0, \tau_{m}\right]$ and $\left[\tau_{m}, \tau_{M}\right]$ are divided into $\eta \geq 2$ and $\lambda \geq 2$ subintervals with equal size, respectively. A new LKF is constructed based on this idea, then new delaydependent stability criteria for time-delay systems are derived by using the free weight matrices method and Jensen's inequality. The proposed criteria can provide less conservative results than some of the existing literature. Numerical examples are given to demonstrate the effectiveness of the proposed method.

\section{Problem Formulation}

Consider the following system

$$
\left\{\begin{array}{l}
\dot{x}(t)=A x(t)+A_{1}(t-d(t)) \\
x(t)=\phi(t), \quad t \in\left[-\tau_{M}, 0\right]
\end{array}\right.
$$

where $x(t) \in \mathbb{R}^{n}$ is the state vector, $A \in \mathbb{R}^{n \times n}, A_{1} \in \mathbb{R}^{n \times n}$ are known real constant matrices; $\phi(t)$ is a given continuous vector-valued initial function and subsection differential on $\left[-\tau_{M}, 0\right] ; d(t)$ is the time-varying delay which satisfies

$$
\tau_{m} \leq d(t) \leq \tau_{M}
$$

where $\tau_{m}, \tau_{M}$ are constants satisfying $0 \leq \tau_{m} \leq \tau_{M}$. The time-varying delay is assumed to be fast-varying (with no restrictions on the delay's derivative).

The following Lemma will be useful throughout this paper.

Lemma 1. (Jensen's inequality) For any given symmetric positive matrix $W>0$, scalars $h_{1}$, $h_{2}$ such that $h_{1} \leq h_{2}$, and vector function $x:\left[h_{1}, h_{2}\right] \rightarrow \mathbb{R}^{n}$, we have:

$$
\left(h_{2}-h_{1}\right) \int_{h_{1}}^{h_{2}} x^{T}(s) W x(s) d s \geq\left(\int_{h_{1}}^{h_{2}} x(s) d s\right)^{T} W\left(\int_{h_{1}}^{h_{2}} x(s) d s\right) .
$$

\section{Main Results}

We first divide the delay interval $\left[0, \tau_{m}\right]$ into $\eta \geq 2$ equally spaced subintervals as in [12], and divide the delay interval $\left[\tau_{m}, \tau_{M}\right]$ into $\lambda \geq 2$ equally spaced subintervals: $\left[\tau_{m}, \tau_{1}\right]$, [i1; $\left.\dot{\Sigma}_{2}\right]$, $\varphi \varphi x,\left[\dot{c}\right.$; 1 ; $\dot{\mathcal{L} M}$ ], where $\tau_{i}=\tau_{m}+i \frac{\tau_{M}-\tau_{m}}{\lambda}(i=1,2, . ., \lambda-1)$. The proposed stability analysis, with $\eta+\lambda$ subintervals, is based on the following LKF candidate: 


$$
V(t)=\sum_{i=1}^{5} V_{i}(t),
$$

where

$$
\begin{aligned}
& V_{1}(t)=x^{T}(t) P x(t), \\
& V_{2}(t)=\int_{t-\tau_{1}}^{t-\tau_{m}} \zeta_{1}^{T}(s) Q \zeta_{1}(s) d s, \\
& V_{3}(t)=\int_{t-\frac{1}{\eta} \tau_{m}}^{t} \zeta_{2}^{T}(s) R \zeta_{2}(s) d s, \\
& V_{4}(t)=\int_{-\tau_{M}}^{0} \int_{t+s}^{t} \dot{x}^{T}(\theta) W_{1} \dot{x}(\theta) d \theta d s, \\
& V_{5}(t)=\int_{-\tau_{M}}^{-\tau_{m}} \int_{t+s}^{t} \dot{x}^{T}(\theta) W_{2} \dot{x}(\theta) d \theta d s,
\end{aligned}
$$

with

$$
\begin{aligned}
& \zeta_{1}(s)=\operatorname{col}\left\{x(s) x\left(s-\tau_{1}+\tau_{m}\right) \quad \cdots \quad x\left(s-\tau_{\lambda-1}+\tau_{m}\right)\right\}, \\
& \zeta_{2}(s)=\operatorname{col}\left\{x(s) x\left(s-\frac{1}{\eta} \tau_{m}\right) \cdots x\left(s-\frac{\eta-1}{\eta} \tau_{m}\right)\right\}, \\
& Q=\left[\begin{array}{ccc}
Q_{11} & \cdots & Q_{1 \lambda} \\
\vdots & \ddots & \vdots \\
* & \cdots & Q_{\lambda \lambda}
\end{array}\right], R=\left[\begin{array}{ccc}
R_{11} & & \\
& \ddots & \\
& & R_{\eta \eta}
\end{array}\right] .
\end{aligned}
$$

One can see that if the conditions

$$
\begin{aligned}
& P>0, W_{1}>0, W_{2}>0, \\
& Q=\left[\begin{array}{ccc}
Q_{11} & \cdots & Q_{1 \lambda} \\
\vdots & \ddots & \vdots \\
* & \cdots & Q_{\lambda \lambda}
\end{array}\right]>0, \\
& R=\operatorname{diag}\left\{R_{11} \cdots R_{\eta \eta}\right\}>0
\end{aligned}
$$

are satisfied, then the $\operatorname{LKF}(3)$ is positive definite.

Remark 1. The analysis method proposed in [12] can reduce considerably the conservativeness for large values of $\tau_{m}$, but the benefits are shortened when the interval $\left[0, \tau_{m}\right]$ is reduced. In this case, the contributions of partitioning the interval $\left[\tau_{m}, \tau_{M}\right]$ are more significant. In [9 12], the interval $\left[\tau_{m}, \tau_{M}\right]$ is simply divided into two equally subintervals, therefore, there is still room for improvement by dividing this interval into more subintervals.

Before presenting the main results, we define $\xi(t)=\operatorname{col}\left\{x(t) x(t-d(t)) x\left(t-\frac{\tau_{m}}{\eta}\right) \cdots\right.$ $\left.x\left(t-\eta \frac{\tau_{m}}{\eta}\right) x\left(t-\tau_{1}\right) \cdots x\left(t-\tau_{\lambda-1}\right) \mathrm{x}\left(\mathrm{t}-\tau_{M}\right)\right\} \quad, \quad$ and $\quad e_{i}(i=1,2, \ldots, \eta+\lambda+2)$, $\hat{e}_{j}(j=1, \ldots, \lambda)$ are block entry matrices. For example, $e_{1}^{T}=\left[\begin{array}{lll}I & 0 & \underbrace{0 \ldots 0}_{\eta+\lambda}\end{array}\right], \hat{e}_{2}^{T}=\left[\begin{array}{lll}0 & I & \underbrace{0 \ldots 0}_{\lambda}\end{array}\right]$.

Theorem 1. For given scalars $\tau_{m}, \tau_{M}, \eta$ and $\lambda$, such that $0<\tau_{m}<\tau_{M}, \eta \geq 2, \lambda \geq 2$, the system (1) is asymptotically stable if there exist matrices $P, W_{1}, W_{2}, Q, R$ in appropriate dimensions satisfying (4)-(6), and free-weighting matrices $N_{a b} \in \mathbb{R}^{n \times n}$ for $a \in\{1, \ldots, \lambda\}$, $b \in\{1,2,3,4\}$, such that the following LMIs hold: 
International Journal of Control and Automation Vol.7, No.4 (2014)

$$
\Psi_{i j}=\left[\begin{array}{cccc}
\Phi+\Omega_{i} & \Upsilon W_{1} & \Upsilon W_{2} & \Gamma_{i j} \\
* & -\frac{1}{\tau_{M}} W_{1} & 0 & 0 \\
* & * & -\frac{1}{h} W_{2} & 0 \\
* & * & * & -\frac{\lambda}{h}\left(W_{1}+W_{2}\right)
\end{array}\right]<0
$$

where

$$
\begin{aligned}
& \Phi=\left[\begin{array}{ccc}
\Phi_{1} & 0 & 0 \\
* & \Phi_{2} & 0 \\
* & * & \Phi_{3}
\end{array}\right], \\
& \Phi_{1}=\left[\begin{array}{cc}
P A+A^{T} P+R_{11} & P A_{1} \\
* & 0
\end{array}\right], \\
& \Phi_{2}=\operatorname{diag}\left\{R_{22}-R_{11} R_{33}-R_{22} \cdots R_{\eta \eta}-R_{(\eta-1)(\eta-1)}\right\}, \\
& \Phi_{3}=\left[\begin{array}{ccccc}
\Pi_{11} & Q_{12} & \cdots & Q_{1 \lambda} & 0 \\
* & \Pi_{22} & \cdots & \Pi_{2 \lambda} & -Q_{1 \lambda} \\
\vdots & \vdots & \ddots & \vdots & \vdots \\
* & * & \cdots & \Pi_{\lambda \lambda} & -Q_{(\lambda-1) \lambda} \\
* & * & \cdots & * & -Q_{\lambda \lambda}
\end{array}\right], \\
& h=\tau_{M}-\tau_{m}, \\
& \Upsilon=e_{1} A^{T}+e_{2} A_{1}^{T}, \\
& \Pi_{m n}=\left\{\begin{array}{l}
Q_{11}-R_{\eta \eta}, m=1, n=1, \\
Q_{m n}-Q_{(m-1)(n-1)}, m, n \in\{2, \ldots, \lambda\}, m \leq n,
\end{array}\right. \\
& \Gamma_{i 1}=e_{\eta+i+1} N_{i 1}+e_{2} N_{i 2}, \\
& \Gamma_{i 2}=e_{2} N_{i 3}+e_{\eta+i+2} N_{i 4} \text {, } \\
& \Omega_{i}=-\frac{\eta}{\tau_{m}}\left(e_{1}-e_{3}\right) W_{1}\left(e_{1}^{T}-e_{3}^{T}\right)-\sum_{l=3}^{\eta+1} \frac{\eta}{\tau_{m}}\left(e_{l}-e_{l+1}\right) W_{1}\left(e_{l}^{T}-e_{l+1}^{T}\right) \\
& -\sum_{k=1, k \neq i}^{\lambda} \frac{\lambda}{h}\left(e_{\eta+k+1}-e_{\eta+k+2}\right)\left(W_{1}+W_{2}\right)\left(e_{\eta+k+1}^{T}-e_{\eta+k+2}^{T}\right) \\
& +\left(e_{\eta+i+1}-e_{2}\right) \Gamma_{i 1}^{T}+\Gamma_{i 1}\left(e_{\eta+i+1}^{T}-e_{2}^{T}\right) \\
& +\left(e_{2}-e_{\eta+i+2}\right) \Gamma_{i 2}^{T}+\Gamma_{i 2}\left(e_{2}^{T}-e_{\eta+i+2}^{T}\right) .
\end{aligned}
$$

Proof. Taking the derivative of the LKF (3) with respect to $t$ along the trajectory of (1) yields

$$
\dot{V}(t)=\sum_{i=1}^{5} \dot{V}_{i}(t)
$$


where

$$
\begin{aligned}
& \dot{V}_{1}(t)=\dot{x}^{T}(t) P x(t)+x^{T}(t) P \dot{x}(t), \\
& \dot{V}_{2}(t)=\zeta_{1}^{T}\left(t-\tau_{m}\right) Q \zeta_{1}\left(t-\tau_{m}\right)-\zeta_{1}^{T}\left(t-\tau_{1}\right) Q \zeta_{1}\left(t-\tau_{1}\right), \\
& \dot{V}_{3}(t)=\zeta_{2}^{T}(t) R \zeta_{2}(t)-\zeta_{2}^{T}\left(t-\frac{1}{\eta} \tau_{m}\right) R \zeta_{2}\left(t-\frac{1}{\eta} \tau_{m}\right), \\
& \dot{V}_{4}(t)=\tau_{M} \dot{x}^{T}(t) W_{1} \dot{x}(t)-\int_{t-\tau_{M}}^{t} \dot{x}^{T}(s) W_{1} \dot{x}(s) d s, \\
& \dot{V}_{5}(t)=h \dot{x}^{T}(t) W_{2} \dot{x}(t)-\int_{t-\tau_{M}}^{t-\tau_{m}} \dot{x}^{T}(s) W_{2} \dot{x}(s) d s .
\end{aligned}
$$

Expanding the integral terms in $\dot{V}_{4}(t)$ and $\dot{V}_{5}(t)$, we have

$$
\begin{aligned}
\dot{V}_{4}(t)+\dot{V}_{5}(t)= & \xi^{T}(t) \Upsilon \Theta \Upsilon^{T} \xi(t)-\int_{t-\tau_{M}}^{t-\tau_{\lambda-1}} f(s) d s-\cdots-\int_{t-\tau_{1}}^{t-\tau_{m}} f(s) d s \\
& -\int_{t-\tau_{m}}^{t-\frac{\eta-1}{\eta} \tau_{m}} g(s) d s-\cdots-\int_{t-\frac{1}{\eta} \tau_{m}}^{t} g(s) d s,
\end{aligned}
$$

where

$$
\begin{aligned}
& \Theta=\tau_{M} W_{1}+h W_{2}, \\
& f(s)=\dot{x}^{T}(s)\left(W_{1}+W_{2}\right) \dot{x}(s), \\
& g(s)=\dot{x}^{T}(s) W_{1} \dot{x}(s) .
\end{aligned}
$$

Consider the case that $d(t) \in\left[\tau_{m}, \tau_{1}\right]$. Using Leibniz-Newton formula, we obtain

$$
\begin{aligned}
-\int_{t-\tau_{1}}^{t-\tau_{m}} f(s) d s= & -\int_{t-\tau_{1}}^{t-d(t)} f(s) d s-\int_{t-d(t)}^{t-\tau_{m}} f(s) d s \\
& +2 \xi^{T}(t) \Gamma_{11}\left[x\left(t-\tau_{m}\right)-x(t-d(t))-\int_{t-d(t)}^{t-\tau_{m}} \dot{x}(s) d s\right] \\
& +2 \xi^{T}(t) \Gamma_{12}\left[x(t-d(t))-x\left(t-\tau_{1}\right)-\int_{t-\tau_{1}}^{t-d(t)} \dot{x}(s) d s\right] \\
\leq & 2 \xi^{T}(t) \Gamma_{11}\left[x\left(t-\tau_{m}\right)-x(t-d(t))\right] \\
& +2 \xi^{T}(t) \Gamma_{12}\left[x(t-d(t))-x\left(t-\tau_{1}\right)\right] \\
& +\left(d(t)-\tau_{m}\right) \xi^{T}(t) \Gamma_{11}\left(W_{1}+W_{2}\right)^{-1} \Gamma_{11}^{T} \xi(t) \\
& +\left(\tau_{1}-d(t)\right) \xi^{T}(t) \Gamma_{12}\left(W_{1}+W_{2}\right)^{-1} \Gamma_{12}^{T} \xi(t) .
\end{aligned}
$$

Then we define $\tau_{0}=\tau_{m}, \tau_{\lambda}=\tau_{M}$, and apply Jensen's inequality (Lemma 1) to other integral terms in (9), which yields

$$
\begin{aligned}
-\int_{t-\tau_{k}}^{t-\tau_{k-1}} f(s) d s \leq & \frac{-\lambda}{\tau_{M}-\tau_{m}}\left(e_{\eta+k+1}-e_{\eta+k+2}\right)\left(W_{1}\right. \\
& \left.+W_{2}\right)\left(e_{\eta+k+1}^{T}-e_{\eta+k+2}^{T}\right), k \in\{2, \ldots, \lambda\}, \\
- & \int_{t-\frac{1}{\eta} \tau_{m}}^{t} g(s) d s \leq \frac{-\eta}{\tau_{m}}\left(e_{1}-e_{3}\right) W_{1}\left(e_{1}^{T}-e_{3}^{T}\right),
\end{aligned}
$$




$$
-\int_{t-\frac{l-1}{\eta} \tau_{m}}^{t-\frac{l-2}{\eta} \tau_{m}} g(s) d s \leq \frac{-\eta}{\tau_{m}}\left(e_{l}-e_{l+1}\right) W_{1}\left(e_{l}^{T}-e_{l+1}^{T}\right), l \in\{3, \ldots, \eta+1\} .
$$

Combing (8)-(13), we have

$$
\dot{V}(t) \leq \xi^{T}(t) \Xi \xi(t)
$$

where

$$
\Xi=\Phi+\Omega_{1}+\Upsilon \Theta \Upsilon^{T}+\Lambda
$$

with $\Phi, \Omega_{1}, \Upsilon$ being defined in (7) and

$$
\Lambda=\left(d(t)-\tau_{m}\right) \Gamma_{11}\left(W_{1}+W_{2}\right)^{-1} \Gamma_{11}^{T}+\left(\tau_{1}-d(t)\right) \Gamma_{12}\left(W_{1}+W_{2}\right)^{-1} \Gamma_{12}^{T} .
$$

If $\Xi<0$, then $\dot{V}(t)<0$ for $d(t) \in\left[\tau_{m}, \tau_{1}\right]$. Note that $\Lambda$ is a convex combination of $\Gamma_{11}\left(W_{1}+W_{2}\right)^{-1} \Gamma_{11}^{T}$ and $\Gamma_{12}\left(W_{1}+W_{2}\right)^{-1} \Gamma_{12}^{T}$ on $d(t) \in\left[\tau_{m}, \tau_{1}\right]$. Therefore, $\Xi<0$ if the following inequalities hold

$$
\begin{aligned}
& \Phi+\Omega_{1}+\Upsilon \Theta \Upsilon^{T}+\frac{h}{\lambda} \Gamma_{11}\left(W_{1}+W_{2}\right)^{-1} \Gamma_{11}^{T}<0, \\
& \Phi+\Omega_{1}+\Upsilon \Theta \Upsilon^{T}+\frac{h}{\lambda} \Gamma_{12}\left(W_{1}+W_{2}\right)^{-1} \Gamma_{12}^{T}<0 .
\end{aligned}
$$

By the Schur complement, (15)(16) are equivalent to $\Psi_{11}<0$ and $\Psi_{12}<0$, respectively.

Similarly, we can derive that if $\Psi_{i 1}<0, \Psi_{i 2}<0$ for $i \in\{2, \ldots, \lambda\}$, then $\dot{V}(t)<0$ for $d(t) \in\left(\tau_{i}, \tau_{i+1}\right]$. Therefore, it is easy to conclude that if (7) hold, then $\dot{V}(t)<0$ for $d(t) \in\left[\tau_{m}, \tau_{M}\right]$. This completes the proof.

Remark 2. Because the delay $d(t)$ varies in the delay range $\left[\tau_{m}, \tau_{M}\right]$, we need to consider the effects of $d(t)$ in each subinterval when we divide this delay range. The integral term $-\int_{t-\tau_{i}}^{t-\tau_{i-1}} f(s) d s$ for $i \in\{1, \ldots, \lambda\}$ in the subinterval $\left[\tau_{i-1}, \tau_{i}\right]$ is fractionized into $-\int_{t-\tau_{i}}^{t-d(t)} f(s) d s$ and $-\int_{t-d(t)}^{t-\tau_{i-1}} x(s) d s$. Then using free weight matrices and the convexity of the matrix function, we avoid the conservatism caused by enlarging $\tau(t)$ to $\tau_{i}$ for each subinterval. Moreover, Jensen's inequality is used to deal with other integral terms which reduces the computational efforts.

Note that the LMIs presented in Theorem 1 are infeasible when $\tau_{m}=0$. In this case we consider a LKF candidate as follows

$$
\hat{V}(t)=V_{1}(t)+V_{2}(t)+V_{4}(t)
$$

where $V_{1}(t), V_{2}(t), V_{4}(t)$ are defined in (3) with $\tau_{m}=0$. Similar to the proof of Theorem 1, one can obtain the following corollary.

Corollary 1. For given scalars $\tau_{M}$ and $\lambda$, such that $\tau_{M}>0, \lambda \geq 2$, the system (1) is asymptotically stable if there exist symmetric matrices $P>0, W_{1}>0, Q$ in appropriate dimensions satisfying (5), and free-weighting matrices $N_{a b} \in \mathbb{R}^{n \times n}$ for $a \in\{1, \ldots, \lambda\}$, $b \in\{1,2,3,4\}$, such that the following LMIs hold: 


$$
\begin{array}{r}
\hat{\Psi}_{i j}=\left[\begin{array}{ccc}
\hat{\Phi}+\hat{\Omega}_{i} & \hat{\Upsilon} W_{1} & \hat{\Gamma}_{i j} \\
* & -\frac{1}{\tau_{M}} W_{1} & 0 \\
* & * & -\frac{\lambda}{\tau_{M}} W_{1}
\end{array}\right]<0 \\
\text { for } i \in\{1,2, \ldots, \lambda\}, \quad j \in\{1,2\},
\end{array}
$$

where

$$
\begin{aligned}
& \hat{\Phi}=\left[\begin{array}{cc}
\hat{\Phi}_{1} & 0 \\
* & \hat{\Phi}_{3}
\end{array}\right], \\
& \hat{\Phi}_{1}=\left[\begin{array}{cc}
P A+A^{T} P & P A_{1} \\
* & 0
\end{array}\right] \text {, } \\
& \hat{\Phi}_{3}=\left[\begin{array}{ccccc}
Q_{11} & Q_{12} & \cdots & Q_{1 \lambda} & 0 \\
* & \Pi_{22} & \cdots & \Pi_{2 \lambda} & -Q_{1 \lambda} \\
\vdots & \vdots & \ddots & \vdots & \vdots \\
* & * & \cdots & \Pi_{\lambda \lambda} & -Q_{(\lambda-1) \lambda} \\
* & * & \cdots & * & -Q_{\lambda \lambda}
\end{array}\right], \\
& \hat{\Upsilon}=\hat{e}_{1} A^{T}+\hat{e}_{2} A_{1}^{T}, \\
& \hat{\Gamma}_{i 1}=\left\{\begin{array}{l}
\hat{e}_{1} N_{11}+\hat{e}_{2} N_{12}, i=1, \\
\hat{e}_{i+1} N_{i 1}+\hat{e}_{2} N_{i 2}, \text { otherwise, }
\end{array}\right. \\
& \hat{\Gamma}_{i 2}=\left\{\begin{array}{l}
\hat{e}_{2} N_{13}+\hat{e}_{3} N_{14}, i=1, \\
\hat{e}_{2} N_{i 3}+\hat{e}_{i+2} N_{i 4}, \text { otherwise, }
\end{array}\right. \\
& \hat{\Omega}_{i}=\left\{\begin{array}{l}
-\sum_{k=2}^{\lambda} \frac{\lambda}{\tau_{M}}\left(\hat{e}_{k+1}-\hat{e}_{k+2}\right) W_{1}\left(\hat{e}_{k+1}^{T}-\hat{e}_{k+2}^{T}\right)+\left(\hat{e}_{1}-\hat{e}_{2}\right) \hat{\Gamma}_{11}^{T}+\hat{\Gamma}_{11}\left(\hat{e}_{1}^{T}-\hat{e}_{2}^{T}\right) \\
+\left(\hat{e}_{2}-\hat{e}_{3}\right) \hat{\Gamma}_{12}^{T}+\hat{\Gamma}_{12}\left(\hat{e}_{2}^{T}-\hat{e}_{3}^{T}\right), i=1, \\
-\frac{\lambda}{\tau_{M}}\left(\hat{e}_{1}-\hat{e}_{3}\right) W_{1}\left(\hat{e}_{1}^{T}-\hat{e}_{3}^{T}\right)-\sum_{k=2, k \neq i}^{\lambda} \frac{\lambda}{\tau_{M}}\left(\hat{e}_{k+1}-\hat{e}_{k+2}\right) W_{1}\left(\hat{e}_{k+1}^{T}-\hat{e}_{k+2}^{T}\right) \\
+\left(\hat{e}_{i+1}-\hat{e}_{2}\right) \hat{\Gamma}_{i 1}^{T}+\hat{\Gamma}_{i 1}\left(\hat{e}_{i+1}^{T}-\hat{e}_{2}^{T}\right)+\left(\hat{e}_{2}-\hat{e}_{i+2}\right) \hat{\Gamma}_{i 2}^{T}+\hat{\Gamma}_{i 2}\left(\hat{e}_{2}^{T}-\hat{e}_{i+2}^{T}\right), \text { otherwise. }
\end{array}\right.
\end{aligned}
$$

\section{Numerical Examples}

In this section, two examples are given to illustrate the less conservatism of our results. Example 1. Consider system (1) for the following parameters:

$$
A=\left[\begin{array}{cc}
-2 & 0 \\
0 & -0.9
\end{array}\right], A_{1}=\left[\begin{array}{cc}
-1 & 0 \\
-1 & -1
\end{array}\right] .
$$

For $\tau_{m}=0$, applying Corollary 1 with different $\lambda$, we obtain the maximal allowable upper bounds of the time-varying delay for which the system remains asymptotically stable. The results are listed in Table 1 . From Table 1 , one can see that dividing the delay interval $\left[0, \tau_{M}\right]$ into $\lambda \geq 3$ subintervals considerably improves the results than the corresponding ones in [13, $14]$. 
Table 1. Maximal $\tau_{M}$ for $\tau_{m}=0$ in example 1

\begin{tabular}{|l|l|}
\hline \multicolumn{1}{|c|}{ Methods } & $\tau_{m}=0$ \\
\hline Theorem $1^{[14]}$ & 1.859 \\
\hline Corollary $2^{[13]}$ & 1.868 \\
\hline Corollary $1^{[13]}$ & 2.118 \\
\hline Corollary $1(\lambda=3)$ & 2.127 \\
\hline Corollary $1(\lambda=4)$ & 2.170 \\
\hline
\end{tabular}

For various $\tau_{m}$, we compare the results obtained by Theorem 1 with those in [12] (see Table 2). It is clear that our method can not only achieve better results than the corresponding ones of [12], but also has fewer decision variables.

Table 2. Maximal $\tau_{M}$ for various $\tau_{m}$ in example 1

\begin{tabular}{|l|l|l|c|}
\hline Methods $\backslash \tau_{m}$ & 1 & 2 & Number of variables \\
\hline Theorem 1 ${ }^{[12]}(\eta=2)$ & 2.217 & 2.751 & 206 \\
\hline Theorem 1 ${ }^{[12]}(\eta=12)$ & 2.230 & 2.779 & 526 \\
\hline Theorem 1 $(\eta=2, \lambda=3)$ & 2.248 & 2.757 & 84 \\
\hline Theorem 1 $(\eta=2, \lambda=4)$ & 2.261 & 2.758 & 115 \\
\hline Theorem 1 $(\eta=12, \lambda=3)$ & 2.261 & 2.784 & 114 \\
\hline Theorem 1 $(\eta=12, \lambda=4)$ & 2.273 & 2.785 & 145 \\
\hline
\end{tabular}

Example 2. Consider system (1) described by

$$
A=\left[\begin{array}{cc}
0 & 1 \\
-1 & -2
\end{array}\right], A_{1}=\left[\begin{array}{cc}
0 & 0 \\
-1 & 1
\end{array}\right] \text {. }
$$

For $\tau_{m}=0$, Table 3 lists the maximal $\tau_{M}$ obtained by Corollary 1 with different $\lambda$. We can see that Corollary 1 can provide less conservative results than [15]. Moreover, Table 4 lists the maximal $\tau_{M}$ for various $\tau_{m}$ obtained by Theorem 1 . From Table 4 , we can see that our results (with $\lambda=2, \eta=2$ or $\lambda=2, \eta=12$ ) coincide with the ones in [12] by taking the same delay interval partition, but are less conservative than the corresponding ones of [12] when the delay interval $\left[\tau_{m}, \tau_{M}\right]$ is divided into $\lambda \geq 3$ subintervals, especially, the improvement is more obvious for small values of $\tau_{m}$.

Table 3. Maximal $\tau_{M}$ for $\tau_{m}=0$ in example 2

\begin{tabular}{|c|c|}
\hline Methods & $\tau_{m}=0$ \\
\hline Theorem 2 $^{[15]}$ & 1.06 \\
\hline Corollary $1(\lambda=2)$ & 1.128 \\
\hline Corollary $1(\lambda=3)$ & 1.150 \\
\hline Corollary $1(\lambda=4)$ & 1.157 \\
\hline
\end{tabular}


Table 4. Maximal $\tau_{M}$ for various $\tau_{m}$ in example 2

\begin{tabular}{|l|l|l|}
\hline Methods $\backslash \tau_{m}$ & 1 & 2 \\
\hline Corollary $7^{[16]}$ & 1.620 & 2.488 \\
\hline Theorem $1^{[12]}(\eta=2)$ & 1.797 & 2.624 \\
\hline${\text { Theorem } 1^{[12]}(\eta=12)}_{1.798}$ & 2.628 \\
\hline Theorem $1(\eta=2, \lambda=2)$ & 1.797 & 2.624 \\
\hline Theorem $1(\eta=2, \lambda=3)$ & 1.804 & 2.626 \\
\hline Theorem $1(\eta=2, \lambda=4)$ & 1.806 & 2.627 \\
\hline Theorem $1(\eta=12, \lambda=2)$ & 1.798 & 2.628 \\
\hline Theorem $1(\eta=12, \lambda=3)$ & 1.806 & 2.631 \\
\hline Theorem $1(\eta=12, \lambda=4)$ & 1.808 & 2.631 \\
\hline
\end{tabular}

\section{Conclusions}

In this paper, a new stability analysis approach for time-delay systems with fastvarying delay has been considered. A general form of the delay-fractioning approach is proposed, which allows further exploitation on the information of both the delay's upper and lower bounds. A new LKF is constructed based on the idea of dividing the delay intervals $\left[0, \tau_{m}\right]$ and $\left[\tau_{m}, \tau_{M}\right]$ into $\eta$ and $\lambda$ equally spaced subintervals, respectively, and delay-dependent stability criteria are derived by using free weight matrices and Jensen's inequality. Some benchmark examples are given to illustrate that the proposed stability criteria are less conservative than some existing results.

\section{References}

[1] J. P. Hespanha, P. Naghshtabrizi and Y. Xu, "A survey of recent results in networked control systems", Proceedings of the IEEE, vol. 95, no. 1, (2007), pp. 138-162.

[2] E. Fridman and U. Shaked, "A descriptor system approach to $H_{\infty}$ control of linear time-delay systems", IEEE Transactions on Automatic Control, vol. 47, no. 2, (2002), pp. 253-270.

[3] E. Fridman, "Stability of linear descriptor systems with delay: a Lyapunov-based approach", Journal of Mathematical Analysis and Applications, vol. 273, no. 1, (2002), pp. 24-44.

[4] Y. He, Q. G. Wang, L. Xie and C. Lin, "Further improvement of free-weighting matrices technique for systems with time-varying delay", IEEE Transactions on Automatic Control, vol. 52, no. 2, (2007), pp. 293299.

[5] H. Shao, "New delay-dependent stability criteria for systems with interval delay", Automatica, vol. 45, no. 3, (2009), pp. 744-749.

[6] K. Liu and E. Fridman, "Wirtinger's inequality and Lyapunov-based sampled-data stabilization", Automatica, vol. 48, no. 1, (2012), pp. $102-108$.

[7] C. Lien, "Delay-dependent stability criteria for uncertain neutral systems with multiple time-varying delays via LMI approach", IEE Proceedings-Control Theory and Application, vol. 152, no. 6, (2005), pp. 707-714.

[8] K. Liu and E. Fridman, "Wirtinger's inequality and Lyapunov-based sampled-data stabilization", Automatica, vol. 48, no. 1, (2012), pp. $102-108$.

[9] X. Jiang and Q. L. Han, "Delay-dependent robust stability for uncertain linear systems with interval timevarying delay", Automatica, vol. 42, no. 6, (2006), pp. 1059-1065.

[10] X. X. Zhang, X. F. Jiang and Q. L. Han, “An Improved Stability Criterion of Networked Control Systems”, Proceedings of the 2010 American Control Conference, Baltimore, MD, USA (2010), June 30-July 2. 
[11] L. F. da Cruz Figueredo, J. Y. Ishihara, "New delay-and-delay-derivative-dependent stability criteria for systems with time-varying delay", Proceedings of the 49th IEEE Conference on Decision and Control, Atlanta, GA, USA, (2010), December 15-17.

[12] L. F. da Cruz Figueredo, J. Y. Ishihara, G. A. Borges and A. Bauchspiess, "A delay-fractioning approach to stability analysis of networked control systems with time-varying delay", Proceedings of the 50th IEEE Conference on Decision and Control and European Control Conference, Orlando, FL, USA. (2011), December 12-15.

[13] E. Fridman, U. Shaked and K. Liu, "New conditions for delay-derivative-dependent stability", Automatica, vol. 45, no. 11, (2009), pp. 2723-2727.

[14] L. Guo, H. Gu, J. Xing and X. He, "Asymptotic and exponential stability of uncertain system with interval delay", Applied Mathematics and Computation, vol. 218, no. 19, (2012), pp. 9997-10006.

[15] P. Park, J. W. Ko and C. Jeong, "Reciprocally convex approach to stability of systems with time-varying delays", Automatica, vol. 47, no. 1, (2011), pp. 235-238.

\section{Authors}

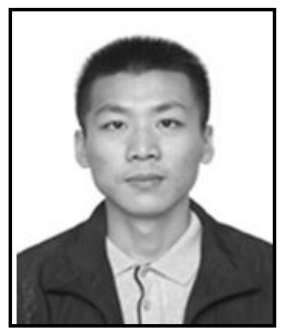

\section{Yuzhi Liu}

He received the M.S. degree in Faculty of Electronic Information and Electrical Engineering from Dalian University of Technology, Dalian, China, in 2011. He is currently working toward the Ph.D. degree in Dalian University of Technology. His research interests include robust control, networked control systems.

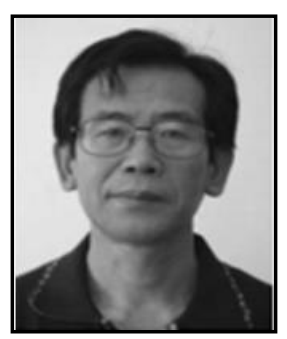

\section{Muguo Li}

He received the B.S. degree in automatic engineering from Dalian University of Technology, Dalian, China, in 1978. He is currently a Professor of the State Key Laboratory of Coastal and Offshore Engineering, Dalian University of Technology. His research interests include networked control systems, image processing, motion control. 